\title{
MOTOR DISORDERS
}

\section{GENOTYPE-PHENOTYPE CORRELATIONS IN ALTERNATING HEMIPLEGIA}

Researchers at the National Center of Neurology and Psychiatry, Kodaira, and multiple centers in Japan, analyze the clinical features and ATP1A3 mutations in 35 Japanese children diagnosed with alternating hemiplegia of childhood (AHC). Gene analysis revealed de novo heterozygous missense mutations of ATP1A3 in 33 patients (7 female and 26 male), Glu815Lys (E815K) in 12 cases (36\%), Asp801Asn (D801N) in 10 (30\%), and other missense mutations in 11 cases. The Glu815Lys genotype is associated with the most severe AHC phenotype. The clinical features associated with these mutations revealed significant differences in the history of neonatal onset, gross motor level, status epilepticus, and respiratory paralysis in the Glu815Lys group compared with the other groups. The E815K mutation group developed abnormal ocular movements or seizures (status) in the first week after birth, and all patients showed very slow development. In contrast, the D801N mutation group had a later and milder onset and course and none showed severe motor deterioration. Other mutation groups also had a milder clinical course. Flunarizine was administered in 31 patients and was discontinued in 13. Seven of the 13 who discontinued flunarizine had either an abrupt or stepwise severe motor deterioration; none of those who continued flunarizine showed severe motor deterioration. (Sasaki M, Ishii A, Saito Y, et al. Genotype-phenotype correlations in alternating hemiplegia of childhood. Neurology 2014 Feb 11;82(6):482-90).

COMMENTARY. Alternating hemiplegia of childhood (AHC) is characterized by recurrent flaccid or dystonic hemiplegia of several minutes or days duration, abnormal ocular movements, involuntary movements, hypotonia, and seizures beginning before 18 months of age. Generally clinical features are sporadic and neuroimaging is normal. De novo mutations of the sodium-potassium-ATPase subunit gene (ATP1A3) are the cause. The time of onset and severity of symptoms of AHC are variable. Patients with early onset tend to have a severe deteriorating course, particularly those with E815K mutation, and sometimes as a sequel to fever and status epilepticus. Flunarizine treatment is recommended in the US and Canada [1], but approval in Japan has been withdrawn [2].

Benign, familial nocturnal alternating hemiplegia is distinguished from AHC by the absence of seizures and motor deterioration. It too responds to flunarizine [3] and is considered a migraine variant [4].

\section{References.}

1. Silver K, Andermann F. Neurology. 1993 Jan;43(1):36-41.

2. Sasaki M, et al. Brain Dev. 2001 Aug;23(5):303-5.

3. Mikati MA, et al. Neurology. 1992 Dec;42(12):2251-7.

4. Sakuragawa N. Brain Dev. 1992 Sep;14(5):283-8. 